\title{
GESTIÓN DEL CORZO EN ZARAGOZA: DE CONSERVACIÓN A CONTROL POBLACIONAL
}

\author{
J. Ferreres ${ }^{1}$, M. A. EsCUdero ${ }^{1}$, E. FERreR ${ }^{2} \&$ J. MARCO $^{1}$ \\ 1 Ebronatura S.L. Calle del Arpa, 6, 50003 Zaragoza. javierferreres@ebronatura.com \\ 2 SODEMASA, Avda. César Augusto, 3, 50004 Zaragoza.eferrer@sodemasa.com
}

ABSTRACT.- As it has happened with other wild ungulates in Europe, the roe deer (Capreolus capreolus) population in Aragon has suffered a considerable expansion during the last two decades. In the Iberian Mountains of Zaragoza (SIZ) this process come along with an increasing sporting interest in the species and the raising of conflicts due to crop damage, mainly on fruit trees and vineyards.

The Government of Aragon is responsible of managing big game populations and fixes the hunting quotas. During the elaboration of hunting plans it has to consider different and even opposite interests and points of view, so availability of reliable population trends it is essential for the management of these populations. It has been developed a scheme for monitoring roe deer population in Aragon based on strip transect counts of pellet groups. This information is complemented by the sightings of roe deer during a fox and lagomorph monitoring scheme based on spotlight counts from car. In the Iberian Mountain System of Zaragoza, between 2005 and 2009, the survey results point that the roe deer abundance has doubled, and the mean density reaches $5.4 \pm 0.33$ roe deer $/ \mathrm{km}^{2}$.

Initially, management plans followed a conservative criterion in order to keep the population growing, with a moderate extraction rate, still hunt and a balanced sex-specific quota. Due to the positive trend of the species and the necessity of reducing damage caused to agriculture, mainly fruit trees, the management goals change to population control and it was decided to increase hunting pressure: hunting season was extended, hunting drives with dogs are authorized and the extraction rate is raised. Although the hunting quota fulfillment is close to $80 \%$, and the number of shot deer has increased from 193 in 2005 to 1.084 in 2009, until now there is no reduction in the population growth.

Keywords: Roe deer; Capreolus capreolus; Management; Hunting quota; Population monitoring; Fecal abundance; Spotlight counts; Aragon. 


\section{J. FERRERES, M. A. ESCUDERO, E. FERRER \& J. MARCO}

RESUMEN.- Al igual que ha ocurrido con otros ungulados en el resto de Europa, durante las dos últimas décadas la población de corzo (Capreolus capreolus) ha experimentado una considerable expansión en Aragón. En el Sistema Ibérico zaragozano (SIZ) este proceso se ha visto acompañado por un incremento del interés cinegético hacia la especie y por una creciente conflictividad asociada a los daños en cultivos de leñosas.

En Aragón es la Administración la responsable de planificar el aprovechamiento cinegético de la caza, asignando los cupos de captura a los cotos de caza. Durante la elaboración de los planes de caza se debe atender a posiciones e intereses muchas veces contrapuestos, por lo que para gestionar estas poblaciones es imprescindible contar con datos objetivos sobre su estado. Para ello se ha desarrollado un plan de seguimiento del corzo en Aragón basado en la realización periódica de transectos de recuento de grupos fecales sobre recorridos fijos. Esta información se complementa con las observaciones de corzo registradas en una red de fareos nocturnos diseñada originalmente para el seguimiento de lagomorfos y zorro. Los datos obtenidos en el SIZ indican que entre 2005 y 2009 la abundancia media de corzo se ha duplicado y que su densidad media alcanza 5,4 $\pm 0,33$ corzos $/ \mathrm{km}^{2}$.

Inicialmente los planes de caza seguían criterios conservadores que permitiesen mantener la población en crecimiento, con una tasa de extracción moderada, un cupo equilibrado entre machos y hembras y una caza selectiva a rececho. Ante la evolución positiva de la especie y la necesidad de atenuar las protestas por daños en cultivos, fundamentalmente frutales, se ha cambiado a una estrategia de control poblacional con un notable incremento de la presión cinegética: se amplía el periodo hábil de caza, se autoriza la caza en batidas y se aumenta la tasa de extracción, aunque se ha mantenido la sex-ratio equilibrada en los cupos. A pesar de que el cumplimiento del cupo se sitúa en torno al 80\%, y se ha pasado de 193 capturas en 2005 a 1.084 en 2009, hasta ahora no se ha conseguido frenar el crecimiento poblacional.

Palabras clave: Corzo; Capreolus capreolus; gestión; seguimiento poblacional; cupos de caza; abundancia fecal; recorridos nocturnos con foco; Aragón.

\section{Introducción}

El incremento de las poblaciones de ungulados silvestres es un fenómeno generalizado en todo el continente europeo y que afecta prácticamente a todas las especies de cérvidos (Apolonio et al., 2010). El corzo es un caso paradigmático, pues durante las últimas décadas además de recuperar zonas en las que había desaparecido debido a la presión humana, se ha extendido de tal manera que probablemente nunca ha tenido una distribución tan amplia como la actual (Linell et al., 1998). Está ocupando hábitats que hasta hace poco no se consideraban adecuados para la especie, como agrosistemas de llanura sin cobertura vegetal, zonas alpinas y ambientes semidesérticos mediterráneos (Linell et al., 1998). 
En Aragón la evolución de la especie ha sido similar a la observada en el resto del continente. A mediados del siglo XIX, la distribución del corzo se limitaba a algunas áreas montañosas de los Pirineos y los sectores más occidentales del Sistema Ibérico. Un siglo más tarde, hacia 1960 su área de distribución se había reducido hasta ocupar un tercio de la estimada en 1850. Desde entonces se produce una rápida expansión y para 1998 se estima que la superficie ocupada por el corzo en Aragón se multiplica por 13 respecto a la de 1960 y cuadruplica la estimada en el siglo XIX hasta alcanzar el 43\% del territorio aragonés (Gortázar et al., 2000). En 2005, Osuna et al., (2006) comprueban que este porcentaje ha subido al 60\%. Este proceso sigue activo y en los últimos años se van sucediendo las citas y observaciones en nuevas localidades (Ferreres et al., 2010).

En el Sistema Ibérico zaragozano (SIZ) la rapidez del proceso ha sido notable. Las primeras observaciones de la especie no aparecen antes de 1985 e inicialmente se limitan a un número reducido de localizaciones en el límite occidental de la provincia (Moncayo y Alto Jalón). Para el año 1999 ocupa prácticamente la totalidad del SIZ de forma que en menos de 15 años el corzo ha colonizado $5.800 \mathrm{~km}^{2}$. Aunque en las fases iniciales la especie selecciona los sectores con características ecológicas más favorables, zonas con precipitaciones más altas y mayor diversidad paisajística, conforme avanza el proceso el corzo ha ido ocupando toda el área (Acevedo et al., 2005).

En esta situación tan dinámica, en la que en pocos años se ha pasado de la ausencia de la especie a una presencia generalizada, la administración aragonesa se ve en la necesidad de desarrollar un programa de seguimiento del corzo que permita obtener unos datos fiables sobre la abundancia y la tendencia poblacional en las que basar su gestión de la especie. Inicialmente desde el Gobierno de Aragón se promovieron diferentes estudios centrados en conocer la distribución y la secuencia de colonización de la especie a nivel local (Marco y Jordán, 1990; Gortázar y Marco, 1998). Más tarde ante el incremento de las poblaciones de corzo se hace necesario un verdadero plan de seguimiento que permita obtener datos fiables de tendencia poblacional y que además, aporte información sobre la densidad absoluta que sirva de base para fijar los cupos de caza. En el diseño de este plan de seguimiento se han considerado condicionantes como la gran extensión de la zona de estudio, las bajas densidades de las poblaciones de corzo, los hábitos forestales de la especie que unidos a su carácter esquivo hacen que la especie sea difícilmente detectable y la disponibilidad de recursos humanos y materiales que, como es habitual, es reducida. Se plantea un seguimiento a gran escala que abarca la totalidad de su área de distribución en Aragón (más de $30.000 \mathrm{~km}^{2}$ ). Atendiendo a características bio-geográficas y extensión se divide el territorio de Aragón en una serie de grandes unidades (Sistema Ibérico Zaragozano, 
Pirineo Oriental etc.) en los que los muestreos se van alternando y repitiendo con periodicidad plurianual (dependiendo de la unidad entre 2 y 4 años). De esta forma se concentra el esfuerzo de muestreo en unos sectores concretos, a la vez que se dejan transcurrir varios años entre dos revisiones del mismo sector. Se consigue así mejorar la precisión de las estimas de abundancia y la fiabilidad de las tendencias aumenta (Smart et al., 2004; Centenera, 2002).

Un plan de gestión de cualquier especie debería contemplar los siguientes aspectos: cuál es el estado de la población, cuales son los objetivos del plan, qué medidas se van a tomar para alcanzar esos objetivos y finalmente, debería establecer un programa de monitorización para determinar en qué medida se han alcanzado esos objetivos (Strickland et al., 1994; Putman, 2003). La decisión de los objetivos de este plan no responde únicamente a argumentos técnicos, y en el fondo es un juicio de valor en el que confluyen aspectos sociales, económicos, biológicos y políticos (Sinclair et al., 2006). En el caso del SIZ, con una considerable ocupación humana del territorio, los objetivos de la gestión de una especie cinegética estarían condicionados por la capacidad de carga social, definida como la densidad máxima que la sociedad considera aceptable en un área determinada (Strickland et al., 1994). Se trataría de un valor subjetivo y variable en el tiempo, que refleja un equilibrio fluido entre los distintos grupos de interés (cazadores, agricultores, etc.).

En este artículo se presentan los resultados obtenidos en el programa de seguimiento del corzo en el Sistema Ibérico Zaragozano, se describe cómo han evolucionado los objetivos de los planes de gestión de la especie, se analiza la efectividad de las medidas adoptadas y finalmente se discuten las perspectivas futuras de su gestión.

\section{Material y métodos}

\section{1. Área de estudio}

El área de estudio se localiza en la provincia de Zaragoza, en el noreste de España ( $42^{\circ} 00^{\prime} 25^{\prime \prime}-40^{\circ} 56^{\prime} 00^{\prime \prime} \mathrm{N}, 2^{\circ} 10^{\prime} 28^{\prime \prime}-0^{\circ} 56^{\prime} 56^{\prime \prime} \mathrm{W}$ ) sobre las estribaciones del Sistema Ibérico. En total son cerca de $5.800 \mathrm{~km}^{2}$ repartidos en un amplio rango altitudinal que abarca desde los 300 a los 2300 m.s.n.m en los pisos climáticos mesomediterráneo y supramediterráneo (Sampietro, 2000). Las precipitaciones son escasas, en la mayor parte del territorio: la precipitación anual se sitúa en torno a los $400 \mathrm{~mm}$ y sólo en las sierras más elevadas supera los 700 (Ascaso \& Cuadrat, 1981). El paisaje está dominado por agrosistemas abiertos, entre los que se intercalan áreas de matorral (Rosmarinus 
officinalis, Quercus coccifera, Juniperus spp, Erica spp) y zonas arboladas, fundamentalmente Quercus ilex y repoblaciones de Pinus spp. Estas manchas boscosas y de matorral generalmente están asociadas a zonas de mayor relieve y cubren $1.299 \mathrm{~km}^{2}$, algo menos del $25 \%$ de la superficie total.

Los datos de abundancia se han obtenido a partir del programa de seguimiento basado en conteos de grupos fecales, complementado con datos de observaciones de corzo recopilados durante la realización de recorridos nocturnos.

\subsection{Estima de densidad por conteo de grupos fecales}

Se han tomado como unidades de muestreo las cuadriculas UTM de 100 $\mathrm{km}^{2}(\mathrm{n}=64)$. A partir del Mapa Forestal de Aragón (Escudero y Franchés, 2005) se ha caracterizado cada una de las cuadrículas desde el punto de vista de la oferta de refugio y la disponibilidad de alimento. En ambientes mediterráneos los hábitat forestados se consideran más adecuados para la especie por ofrecer simultáneamente alimentación y refugio (Acevedo et al., 2005). Así, las teselas que presentan árboles o matorral de más de 1,5 m de altura se han considerado favorables para el corzo y el resto desfavorables.

Para cada cuadrícula se seleccionó sobre el Mapa Forestal de Aragón un punto representativo de la disponibilidad de hábitat favorable para el corzo. En una primera fase del estudio en 1999, en cada punto de muestreo se realizó un recorrido de $2000 \mathrm{~m}$ para localizar indicios de presencia de la especie: grupos fecales, escodaduras, huellas, encames etc. Con estos datos, además de determinar la presencia o ausencia de corzo, se obtuvo un índice kilométrico de abundancia (IKA) a partir del número de grupos fecales de corzo detectados por cada kilómetro de transecto realizado (Mayle et al., 2000). Se consideró grupo de heces cuando seis o más excrementos parecían producto de la misma defecación. Este índice es una estima de abundancia relativa que, aunque permite estimaciones locales de la tendencia poblacional, al no considerar la influencia del medio sobre la detectabilidad de los grupos fecales, no sería útil para hacer comparaciones entre poblaciones (Cederlund et al., 1998). El IKA es un índice lineal que no permite estimar la densidad absoluta de grupos fecales y por tanto, tampoco la densidad poblacional absoluta (Mayle et al., 1999).

Posteriormente se diseñó una metodología que permitiera valorar la superficie revisada a lo largo del transecto, para poder estimar la densidad de grupos fecales y calcular la densidad de corzo. La metodología empleada, transectos de anchura de banda variable, es una adaptación del recuento de excrementos en transectos en banda, en el que se contabilizan los grupos feca- 
les que se detectan en una banda de anchura fija a cada lado de la línea de progresión (Mayle et al., 1999). En esta adaptación, no se pretende hacer una revisión exhaustiva de la totalidad de la banda en la que se hace el recuento de grupos fecales sino que, para ganar rapidez, únicamente se cuentan los grupos fecales dentro del ancho de banda que se asume se puede revisar sin ralentizar el proceso. En función de la cobertura vegetal se va asignando a cada tramo del transecto $(10 \mathrm{~m})$ un ancho de banda estandarizado en clases de 0, 0,5, 1 ó $2 \mathrm{~m}$, basándose en experiencias previas sobre detectabilidad de los grupos fecales. Se trataría de un transecto en banda en el que el ancho de banda es variable en cada tramo de forma que la superficie revisada es estimada subjetivamente y presenta una relación coste-resultados superior a otros métodos similares. Una descripción más completa, junto a una comparativa frente a otros métodos basados en recuentos de grupos fecales, se puede encontrar en Acevedo et al. (2010).

La superficie total revisada en un transecto sería:

$$
\mathrm{S}=\sum \mathrm{b}_{\mathrm{i}} \times 10
$$

donde " $\mathrm{b}$ " es el ancho de banda de cada tramo de $10 \mathrm{~m}$.

A partir de este dato y conociendo la tasa de persistencia de los grupos fecales y la tasa de defecación se calcula la densidad de corzo (Mayle et al., 1999):

$$
\mathrm{D}=\mathrm{gf} / \mathrm{S} \times \mathrm{td} \times \mathrm{pp}
$$

Donde $\mathrm{S}$ es la superficie revisada, gf es el número de grupos fecales detectados, td es la tasa de defecación (20 g.f. / día, Mayle et al.,1999), y pp el periodo de persistencia, aplicado en nuestro caso, 135 días. Este último valor se obtuvo a partir de una experiencia previa realizada en el Parque Natural del Moncayo (SIZ) en la que se estimó una tasa de desaparición conjunta para un amplio abanico de hábitats, desde coscojales a hayedos (Escudero et al., 2007). Es un valor promedio que se sitúa dentro de los márgenes estimados en ambientes mediterráneos en Grecia, entre 115 y 164 días según tipo de hábitat (Tsaparis et al., 2009), y es inferior a los calculados en el centro de Portugal en un clima con mayor influencia atlántica, entre 151 y 297 días (Carvalho, 2007). Sin embargo, según la estación, se han citado valores entre 26 y 118 en Polonia (Aulak y Babinska, 1990), y dentro del contexto del Reino Unido, entre 64 y 165 días en áreas con menores precipitaciones, y entre 98 y 537 en áreas más lluviosas (Mayle et al., 1999).

Sobre los puntos seleccionados en 1999 se han realizado muestreos basados en transectos de anchura de banda variable que se repiten con periodici- 
dad bienal a partir de 2005 (hasta ahora tres campañas: 2005, 2007 y 2009). En 2005 se rediseñaron los transectos originales de forma que atravesasen áreas representativas del hábitat de la zona, evitando itinerarios paralelos a arroyos, sendas, linderos y otras zonas que puedan influir sobre la abundancia de corzos (Mayle et al., 1999). En cada punto de muestreo se fijaron dos transectos de $700 \mathrm{~m}$ y se georreferenciaron para facilitar su repetición en el tiempo y evitar la variabilidad de los resultados asociada a efectos topográficos. Los muestreos se realizaron en dos periodos: mayo-junio y octubre-noviembre. Para reducir un posible efecto estacional sobre cobertura vegetal, la tasa de persistencia de los grupos fecales o la tasa de defecación, las revisiones de cada punto se realizaron siempre en la misma estación. Con el fin de minimizar el efecto observador, que podría ser importante ya que existe una componente subjetiva en la valoración del ancho de banda, todas las revisiones se han realizado por un solo equipo formado por los mismos tres observadores (M. A. Escudero, E .Ferrer y J. Ferreres), coautores de este trabajo.

\subsection{Estima de la abundancia a partir de avistamientos nocturnos}

La información obtenida a partir de los recorridos de abundancia fecal, se complementa con los datos de avistamientos ocasionales de corzo durante la realización de recorridos, en el marco del Plan de Monitorización de Mamíferos de Aragón (PMMA) (Gortázar et al., 1994). Este plan está diseñado para monitorizar a gran escala las poblaciones de mesomamíferos, fundamentalmente lagomorfos (conejo Oryctolagus cuniculus, liebre europea Lepus europaeus, liebre ibérica Lepus granatensis y zorro Vulpes vulpes) y consiste en una red de 60 localidades repartidas por todo el territorio, en las que se realizan conteos nocturnos desde vehículo equipado con faro adicional. Los itinerarios son recorridos fijos que discurren por pistas agrícolas en agrosistemas abiertos, tienen una longitud aproximada de $30 \mathrm{~km}$ y se repiten dos veces al mes entre enero y abril (8 repeticiones anuales con un total de $240 \mathrm{~km}$ ) por el mismo equipo, formado por dos Agentes de Protección de la Naturaleza del Gobierno de Aragón. El faro de $100 \mathrm{~W}$ es manejado por el copiloto que barre de manera sistemática con el haz de luz un arco de $270^{\circ}$ en torno al vehículo, y cuando es necesario la identificación de los animales se confirma mediante el uso de prismáticos $8 \times 42$. Los recorridos se realizan con periodicidad anual, lo que posibilita el cálculo eficaz de los índices de abundancia relativa y las tendencias demográficas de cada especie (Williams et al., 2007, Gortázar et al., 2007). Durante los recorridos, además de las especies diana se anotan todas las observaciones de otras especies de interés, entre ellas el corzo. 
Se han recopilado las observaciones de corzo en los 12 puntos de monitorización localizados en el área de estudio. A partir del número de corzos avistados y la longitud total recorrida en los transectos se ha calculado un índice kilométrico de abundancia (IKA) expresado en corzos $/ 100 \mathrm{~km}$ (Acevedo et al., 2008). Los datos se han agrupado por bienios.

\subsection{Estadísticas cinegéticas}

Las estadísticas cinegéticas se han elaborado a partir de los datos suministrados por el Instituto Aragonés de Gestión Ambiental del Gobierno de Aragón. Los cupos son los asignados por la Dirección General de Desarrollo Sostenible y Biodiversidad del Gobierno de Aragón, mientras que los resultados se obtienen a partir de las declaraciones de resultados que los titulares de los cotos de caza comunican a la administración al finalizar la temporada de caza. Los objetivos de gestión y tasas de extracción se han obtenido de informes internos del Gobierno de Aragón sobre la gestión del corzo (Ferreres et al., 2010).

\subsection{Análisis de los datos}

Se han utilizado tests no paramétricos (prueba de Kruskal-Wallis) para hacer comparaciones entre periodos y regresiones lineales para estudiar la tendencia poblacional. Con el fin de unificar periodos (datos disponibles de cupos, capturas, datos de abundancia) las tendencias poblacionales se han calculado con los datos disponibles a partir de 1999.

\section{Resultados}

\subsection{Transectos de abundancia fecal}

Se han realizado transectos en 64 puntos de muestreo. Las cuatro campañas de trabajo de campo entre 1999 y 2009, muestran el incremento poblacional de la especie (Tabla 1). En un periodo de 6 años, entre 1999 y 2005, el corzo pasa de ser detectado en la mitad de los puntos de muestreo a más del $90 \%$ de puntos positivos, y dos años más tarde se alcanza el 100\% de puntos positivos. Los índices de abundancia confirman un continuo crecimiento poblacional, tanto inicialmente, entre 1999 y 2005, como en los últimos años, cuando los muestreos se realizan con periodicidad bienal (Figura 1). 
GESTIÓN DEL CORZO EN ZARAGOZA: DE CONSERVACIÓN A CONTROL POBLACIONAL

Tabla 1. Evolución de los cupos, capturas y abundancia de corzo estimada a partir de transectos de abundancia fecal. Se indica el factor de crecimiento observado entre los distintos periodos $\left(\mathrm{N}_{\text {final }} / \mathrm{N}_{\text {inicial }}\right)$.

Table 1. Evolution of roe deer hunting quota, hunting bag and abundance estimated from fecal strip counts. The growth factor is also indicated.

\begin{tabular}{|c|c|c|c|c|c|c|c|}
\hline & 1999 & 2005 & 2007 & 2009 & $\begin{array}{c}\text { Increment } \\
\text { o 1999-2009 }\end{array}$ & $\begin{array}{c}\text { Increment } \\
\text { o 1999-2005 }\end{array}$ & $\begin{array}{c}\text { Increment } \\
\text { o 2005-2009 }\end{array}$ \\
\hline $\begin{array}{c}\text { \% puntos muestreo } \\
\text { positivos }\end{array}$ & 54,6 & 93,9 & 100 & 100 & $\times 1,8$ & $\times 1,7$ & $\times 1,1$ \\
IKA fecal (g.f./km) & 0,8 & 12,3 & 17,5 & 21,5 & $\times 26,9$ & $\times 15,4$ & $\times 1,7$ \\
$\begin{array}{c}\text { Densidad } \\
\left.\text { (corzos/ km }{ }^{2}\right)\end{array}$ & - & 2,6 & 3,9 & 5,4 & - & - & $\times 2,1$ \\
Cupo total (corzos) & 46 & 258 & 700 & 1.272 & $\times 27,5$ & $\times 11,2$ & $\times 4,9$ \\
Capturas (corzos) & 23 & 193 & 533 & 1.084 & $\times 47,1$ & $\times 8,4$ & $\times 5,6$ \\
\hline
\end{tabular}

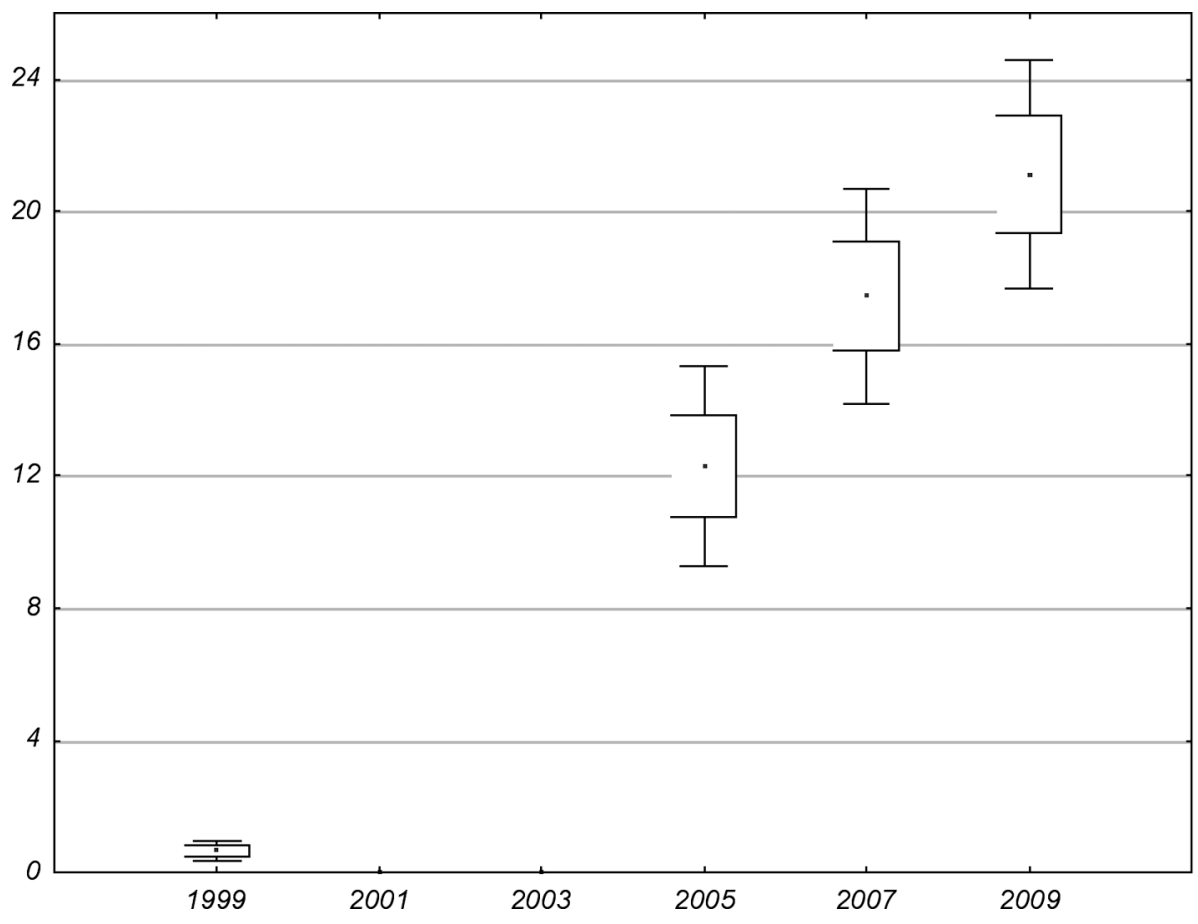

Figura 1. Abundancia de corzo en el Sistema Ibérico Zaragozano (grupos fecales/ $1000 \mathrm{~m}$ de transecto), (media, media $\pm \mathrm{EE}$, media $\pm 1,96^{*} \mathrm{EE}$ ). La abundancia se ha estimado a partir de transectos a pie realizados en 64 puntos de muestreo fijos.

Figure 1. Roe deer abundance in the SIZ (pellet group $/ 1000 m$ ), (median, median $\pm S E$, median $\pm 1,96 * S E$ ). Abundance is estimated from fecal standing crop transects in 64 fixed points. 
Este incremento de la abundancia se refleja en un incremento de las densidades estimadas que pasan de 2,6 $\pm 0,29$ corzos $/ \mathrm{km}^{2}$ en 2005 a 3,9 $\pm 0,35$ en 2007 y alcanza 5,4 0,33 en 2009 (no hay estimas de densidad en 1999), con diferencias interanuales significativas $(\mathrm{KW}, \mathrm{H}=27,95 ; \mathrm{p}=0,000)$. La tendencia poblacional es positiva, con una tasa anual de crecimiento del $15,8 \%$, $\left(\mathrm{R}^{2}\right.$ $=0,997 ; \mathrm{p}=0,002 ; \beta=2,07$ ).

\subsection{Avistamientos nocturnos}

Los datos obtenidos a partir de observaciones de corzo durante los recorridos nocturnos del PMMA reflejan la expansión de la población de corzo en el SIZ, apreciable tanto por el aumento del área de distribución como en la abundancia. Hasta 1999 sólo se habían observado corzos en dos de las 12 localidades donde se realizan muestreos, desde entonces se va detectando la especie en un número creciente de localidades, 5 en 2001, 8 en 2004 y en 2007 ya se cita la especie en todas las localidades prospectadas. La evolución de la abundancia sigue una tendencia similar (Figura 2), valores de IKA muy redu-

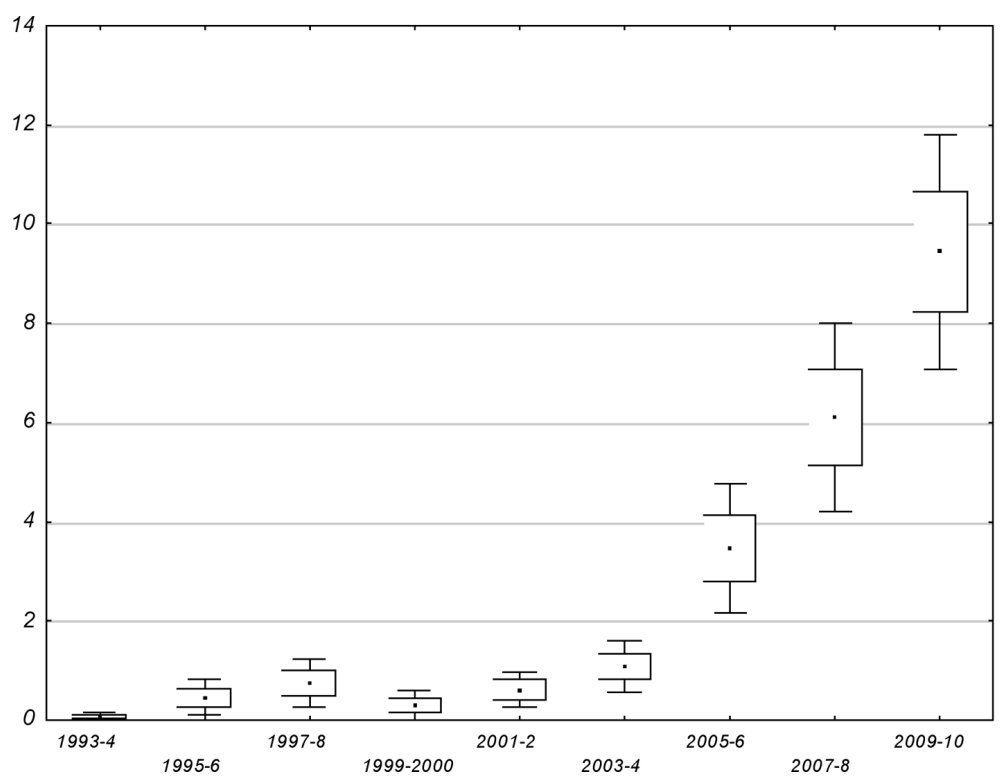

Figura 2. Abundancia de corzo en el SIZ (corzos $/ 100 \mathrm{~km}$ ), (media, media $\pm \mathrm{EE}$, media $\pm 1,96^{*} \mathrm{EE}$ ), estimada a partir de recorridos nocturnos en vehículo en 12 localidades $(180 \mathrm{~km}$ por localidad y año). Figure 2. Roe deer abundance in the SIZ (deer $/ 100 \mathrm{~km}$ ), (median, median \pm SE, median $\pm 1,96^{*} \mathrm{SE}$ ).

Abundance is estimated from spotlight counts in 12 localities (180 km per locality and year). 
GESTIÓN DEL CORZO EN ZARAGOZA: DE CONSERVACIÓN A CONTROL POBLACIONAL

cidos, por debajo de 0,5 corzos / $100 \mathrm{~km}$ en los años 90, y a partir de entonces se dispara, alcanzando los 9 corzos/100km en 2009 (1999: 0,3 0,14 corzos / $100 \mathrm{~km}$; 2005: 3,5 \pm 0,67; 2007: 6,1 $\pm 0,97 ; 2009: 9,4 \pm 1,21)$. Diferencias entre periodos significativas $(\mathrm{KW}, \mathrm{H}=215,98, \mathrm{p}=0,000)$. La tendencia poblacional es positiva, con una tasa anual de crecimiento entre 2005 y 2009 del 21,9 $\%,\left(\mathrm{R}^{2}=0,896, \mathrm{p}=0,004 ; \beta=0,896\right)$.

\subsection{Estadísticas cinegéticas}

Los cupos y las capturas de corzo en el SIZ han seguido un patrón similar al que muestra la evolución de la abundancia de la especie (Figura 3).

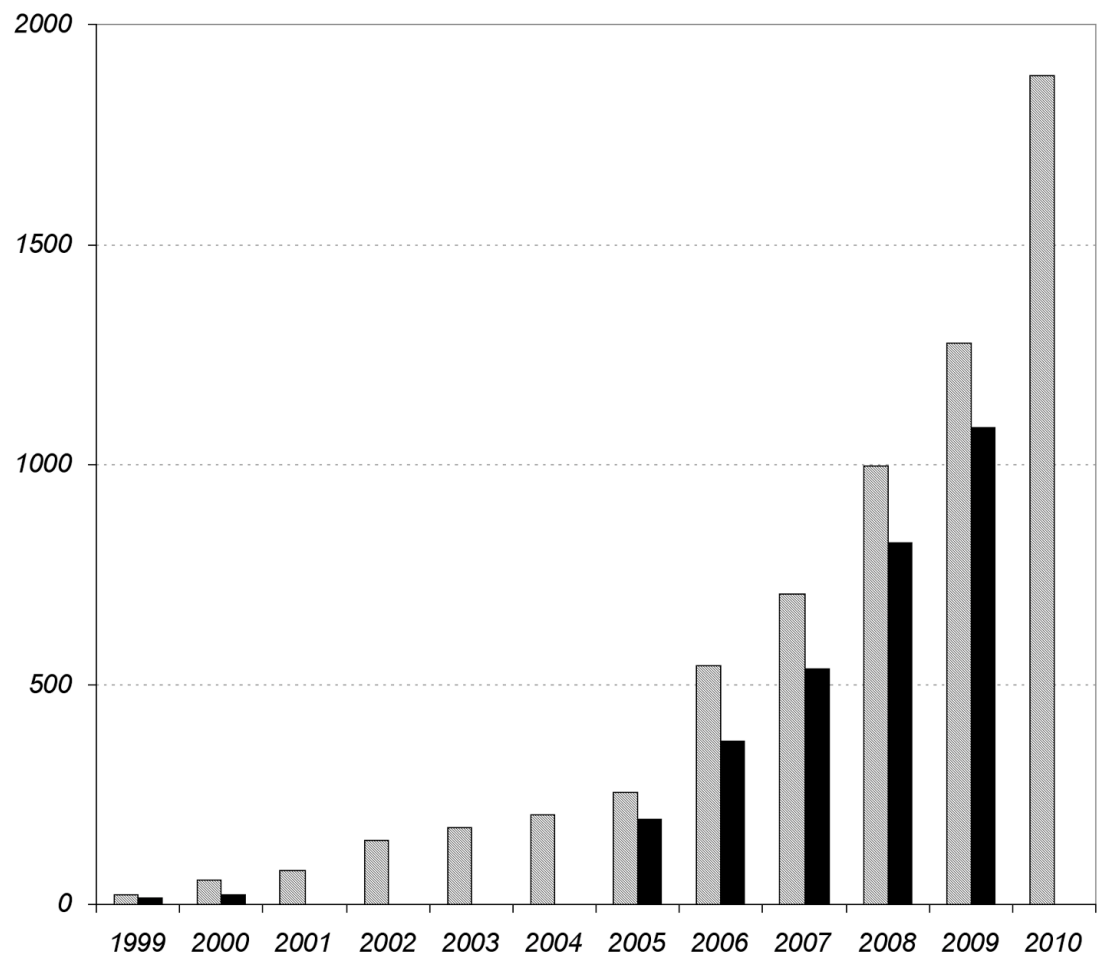

Figura 3. Cupos de corzo en el Sistema Ibérico Zaragozano (SIZ). Se indica en gris el cupo autorizado por el Gobierno de Aragón y en negro las capturas declaradas por los cazadores (no hay datos disponibles entre 2001 y 2004).

Figure 3. Hunting quotas in the SIZ. In grey the hunting quotas set by the Aragon Government, in black the hunting bags reported by hunters (no data available in the 2001-2004 period). 
Entre 1999 y 2005, los cupos asignados y las capturas son reducidos. En esta fase inicial del aprovechamiento cinegético, por precaución y debido a la falta de datos objetivos sobre los que calcular los cupos, éstos se asignan siguiendo criterios conservadores, las capturas no llegan a 200 ejemplares por temporada. A partir de 2005 se obtienen las primeras estimaciones de densidad, con valores más altos que los supuestos hasta entonces, lo que permite incrementar considerablemente los cupos. Los sucesivos estudios van mostrando un crecimiento poblacional sostenido entre 2005 y 2009, lo que unido a una creciente conflictividad motivada por daños agrícolas, hace que se decida incrementar la presión cinegética sobre la especie: se amplía el periodo hábil de caza hasta 11 meses, se favorece la caza en batidas, y especialmente en las dos últimas temporadas de caza se incrementan los cupos de caza sustancialmente, con una tasa de extracción que supone el 30\% de la población total estimada y que en algunos sectores donde la presencia de cultivos de frutal es mayor, se incrementa hasta superar el $40 \%$ de la población (Ferreres et al., 2010). Mientras que entre 2005 y 2009 la densidad se multiplica por dos, en este mismo periodo los cupos autorizados se multiplican por 5. En el caso de las capturas declaradas el incremento es aún mayor, y en 2009 se superan los 1.000 ejemplares cazados. El grado de cumplimiento de los cupos de extracción ha ido creciendo y se situaría en torno al 80\% (Tabla 1). Según las declaraciones de resultados de caza el 52\% de los 3.189 animales abatidos son machos.

\section{Discusión}

Los muestreos de abundancia fecal basados en transectos de anchura variable están basados en apreciaciones subjetivas del ancho de banda y presentan como gran inconveniente su susceptibilidad a errores debidos al efecto observador (Acevedo et al., 2010). Por otro lado, posibles variaciones de la tasa de defecación ligadas a factores como la alimentación, densidad, estructura poblacional y variaciones estacionales de la calidad de la dieta podrían afectar a la validez de los cálculos de la densidad de la población a partir de la densidad de grupos fecales, por lo que, en el mejor de los casos, los conteos de grupos fecales deberían ser usados como un indicador de cambios en la densidad a nivel local (Cederlund et al., 1998). Sin embargo, el diseño del seguimiento del corzo en Aragón, en el que se repiten los mismos transectos en las mismas fechas y por los mismos observadores, reduce al mínimo la variabilidad asociada a efectos locales, estacionales o del observador. Para completar los datos de abundancia obtenidos a partir del seguimiento de corzo mediante grupos fecales, se ha estimado la evolución de la población de 
corzo a partir de un índice kilométrico de avistamientos (IKA) durante recorridos nocturnos en automóvil. Aunque diversos autores desaconsejan este método para estimar tendencias poblacionales de cérvidos debido a su baja fiabilidad (Mayle et al., 1999; Cederlund et al., 1998; Van Laere et al., 1999), otros autores defienden su utilidad para el seguimiento de las poblaciones de ciervo en ambientes mediterráneos (Acevedo et al., 2008). El hecho de que dos métodos completamente distintos, uno indirecto basado en indicios de presencia y otro directo, muestren resultados coincidentes, confirmaría la validez de la tendencia observada. Por otro lado, el esfuerzo de muestreo empleado en el programa de seguimiento, ha sido suficiente para detectar un incremento significativo en la abundancia de corzo.

Los valores de densidad obtenidos deben interpretarse como una aproximación general a la densidad media, ya que en el cálculo se ha empleado un único valor para el periodo de persistencia en todos los puntos de muestreo, y no se han empleado tasas de desaparición específicas para los principales tipos de vegetación presentes en el área de estudio, lo que habría mejorado la exactitud de las estimas (Mayle et al., 1999; Putman, 2003; Campbell et al., 2004). Como ya se ha comentado, se trata de un valor promedio (135 días) estimado a partir de una experiencia previa en una parte del área de estudio, y se sitúa dentro de los márgenes estimados o es inferior a los calculados por diversos autores en ambientes mediterráneos (Tsaparis et al., 2009; Carvalho, 2007). Con estos datos sería esperable que los valores de densidad estimados fuesen cercanos a los reales o se produjese una ligera sobrestima. La densidad estimada en 2009, en torno a 5,5 corzos $/ \mathrm{km}^{2}$ es similar a las de otras poblaciones del interior de la Península Ibérica, 6,5 en Guadalajara (Burón et al., 2005) y 5,6 en Castilla - La Mancha (Acevedo et al., 2010). Es la más alta observada en Aragón: entre 0,5 y 1,7 en Teruel, y entre 1,8 y 2,3 en Huesca (Ferreres et al., 2010) y supera ampliamente la estimada en sectores con mayor influencia eurosiberiana como las Reservas de Caza del Pirineo aragonés, 2,4 corzos $/ \mathrm{km}^{2}$ (Prada et al., 2010). Este hecho contrasta con los valores observados en otros puntos de la Península Ibérica y Francia, en donde se describen abundancias más bajas en los sectores con mayor carácter mediterráneo (Aragón et al., 1995, Virgós y Tellería 1998, Gaudin et al., 1997, Acevedo et al., 2005).

En esta situación de expansión poblacional y densidades moderadas, se planteó la gestión del corzo en el SIZ. Existen cuatro posibles opciones al gestionar una especie: fomentar el incremento poblacional (conservación), perseguir una reducción en su densidad (control), realizar una extracción sostenible (explotación) y no tomar ninguna medida pero monitorizar su evolución (vigilancia) (Sinclair et al., 2006). La gestión del corzo en el SIZ ha pasado por estas cuatro opciones. En la fase inicial del proceso simplemente se vigilaba la 
evolución de la especie y a partir de 1999 se decidió comenzar el aprovechamiento cinegético en unas condiciones con las que se perseguía favorecer la expansión de las poblaciones. Posteriormente se fue comprobando que la abundancia del corzo aumentaba y surgió una considerable problemática asociada a daños en los cultivos de frutales (Ferreres et al., 2010). En este contexto el objetivo de la gestión pasa a ser el control del crecimiento poblacional mediante un incremento de la presión cinegética. Aunque el aumento en los cupos ha sido notable durante los últimos años, muy superior al de la abundancia, hasta ahora no ha sido suficiente para contener el crecimiento poblacional.

El éxito de un plan de gestión se puede valorar como el grado de consecución de los objetivos marcados (Sinclair et al., 2006). Bajo este punto de vista, los planes de gestión del corzo en el SIZ fueron adecuados mientras se persiguió favorecer la expansión de la especie y el fomento de su aprovechamiento cinegético. Sin embargo, cuando los objetivos cambiaron y se intentó estabilizar el tamaño poblacional para reducir el nivel de daños, los resultados no están siendo los deseados. Al igual que está ocurriendo en muchas zonas de Europa, los planes de gestión de los ungulados están encontrando muchos problemas a la hora de controlar sus poblaciones (Apollonio et al., 2010). En una población en expansión la tasa anual de crecimiento teórica se situaría en torno al 35 \% (Sáez de Buruaga, 1997; Boisaubert y Muron, 1997), por lo que la tasa de crecimiento observada en el SIZ, el 20\%, sería mayor que la esperable tras aplicar una tasa de extracción cercana al 30\%. Diversos factores pueden estar influyendo en esta situación. En primer lugar, la extracción podría ser inferior a la prevista en el plan de caza, ya que según declaran los cazadores, el grado de cumplimiento del cupo está en el $80 \%$. Por otra parte, parece existir cierta incidencia de caza furtiva, principalmente sobre machos y posiblemente se están declarando capturas que en realidad no se han producido, especialmente en el caso de hembras, lo que provocaría un desequilibrio en la sex-ratio, y que la población aumentara con mayor rapidez (Saéz de Buruaga, 1997; Putman, 2003). Finalmente no es descartable que se esté subestimando el tamaño poblacional, pues se desconoce la exactitud de la metodología que se está empleando para estimar la densidad (Acevedo et al., 2010) y tampoco se ha cuantificado el número de corzos fuera de las áreas favorables, ya que aunque la densidad pueda ser baja, estos hábitats suponen el $75 \%$ de la superficie total.

Para controlar la población de corzo probablemente sea necesario incrementar aún más la presión cinegética sobre la especie. Si actualmente el grado de cumplimiento del cupo se sitúa en torno al $80 \%$, y viendo la evolución de otras especies de ungulados en Aragón (ver Marco et al., 2011 en este mismo número), parece problemático conseguir frenar la tendencia positiva de la 
población sin la adopción de medidas drásticas como podría ser la autorización de la caza sin cupos. Estas medidas circunscritas a los sectores más conflictivos deberían ser acompañadas por un seguimiento riguroso, tanto de evolución de las poblaciones como de los daños agrícolas. Aunque hasta ahora se han marcado los objetivos de gestión influidos por la conflictividad asociada a los daños y no por la valoración objetiva de los daños, sería conveniente desarrollar un sistema de recogida de datos que permita cuantificar su importancia real y la efectividad de las medidas de prevención adoptadas. En este sistema se debería incluir también los datos de accidentes de tráfico con corzos.

Además de mantener el programa actual de seguimiento del corzo basado en indicios de presencia, parece necesario completar esta información con datos fiables sobre la estructura poblacional, basada en un número suficiente de observaciones, que sea representativo del conjunto del área de estudio y especialmente en los sectores donde se autorice la caza sin cupo, ya que este tipo de caza podría desequilibrar fuertemente la sex-ratio hacia las hembras, reduciendo la eficacia de las medidas de control adoptadas.

\section{Referencias}

Aragón, S., Braza, F. \& San José, C., 1995. Socioeconomic, physiognomic and climatic factors determining the distribution pattern of roe deer Capreolus capreolus in Spain, Acta Theriologica, 40: 37-43.

Acevedo, P., Delibes-Mateos, M., Escudero, M. A., Vicente, J., Marco, J. \& Gortázar, C., 2005. Environmental constrains in the colonization sequence of roe deer (Capreolus capreolus Linnaeus, 1785) across the Iberian Mountains, Spain. Journal of Biogeography, 32: 1671-1680.

Acevedo P., Ruiz-Fons, F., Vicente, J., Reyes-García, A.R., Alzaga, V. \& Gortázar, C., 2008. Estimating red deer abundance in a wide range of management situations in Mediterranean habitats. Journal of Zoology, 276: 37-47.

Acevedo P., Ferreres, J., Jaroso, R., Durán, M., Escudero, M.A., Marco, J. \& Gortázar, C., 2010. Estimating roe deer abundance for pellet groups counts in Spain: an assessment of methods suitable for Mediterranean woodlands. Ecological Indicators, 10: 1226-1230.

Apollonio M., Anderson, R. \& Putman, R., 2010. European Ungulates and their management in the $21^{\text {st }}$ century. Cambridge University Press, $618 \mathrm{pp}$. Cambridge.

Ascaso, A. \& Cuadrat, J.M., 1981. Geografía de Aragón. T.I. Guara Ed. 275 pp. Zaragoza. 
Aulak, W. \& Babinska-Werka, J., 1990. Estimation of roe deer density based on the abundance and rate of disapperance of their faeces from de forest. Actha Theriologica, 35 (1-2): 111-120.

Boisaubert, B. \& Mouron, B., 1997. La situation du chevreuil en France. Bulletin Mensuel de l'Office National de la Chasse, 218 : 22-25.

Burón, D., Fernández, L., Martínez, A. L., López, J. C. \& Fernández-Mellado, R., 2005. Status of roe deer population in Northen Guadalajara (Spain). Acta of the $7^{\text {th }}$ European Roe Deer Meeting. Jerez (España).

Campbell, D., Swanson, J. \& Sales, J., 2004. Comparing the precission and cost-effectiveness of faecal pellet group count methods. Journal of Applied Ecology, 41: 1185-1196.

Carvalho, P., 2007. Ecologia de uma população reintroduzida de corços (Capreolus capreolus L.). Tese de Mestrado em Ecologia. Universidade de Coimbra. Coimbra. Portugal.

Cederlund, G., Bergovist, J., Kjellander, P, Gill, R., Gaillard, J. M., Boisuabert, B., Ballon, P. \& Duncan, P., 1998. Managing roe deer and their impact on the enviroment: maximising the net benefits to society. The european roe deer: the biology of success. (ed. by R. Andersen, P. Duncan and J.D.C. Linnell), 338-372. Scandinavian University Press, Oslo (Noruega).

Centenera, R., 2002. Consideraciones en torno a la caza en la conservación del corzo.

II Simposio sobre el corzo en España. Asociación del Corzo Español.195-204. Luarca. España.

Escudero, M. A., Ferreres, J. \& Marco, J., 2007. Situación de la población de corzo en el Parque Natural del Moncayo. Informe interno del Gobierno de Aragón.

Escudero, O. \& Franchés, M. J., 2005. Memoria de síntesis del Mapa Forestal de Aragón. Serie Difusión $\mathrm{n}^{\circ}$ 11, Consejo de Protección de la Naturaleza 119 pp, Zaragoza.

Ferreres, J., Marco, J., Escudero M. A., Labarta, J. L. \& Monrabal, L., 2010. Propuesta de plan de aprovechamiento cinegético del corzo para las temporadas 2005-2011. Informes internos del Gobierno de Aragón.

Gaudin, J.C., Maillard, J.M., \& Gailiard, G., 1997. Colonisation par le Chevreuil (Capreolus capreolus) de la zone méditerrannéenne française. Rev Ecol. (Terre Vie), 52: 113-122.

Gortázar, C., Marco, J. \& Martín, M., 1994. Seguimiento del Plan de Monitorización de Abundancias de Mamíferos. Factores ecológicos limitantes y dinámica de las poblaciones vulpinas. Informe interno del Gobierno de Aragón.

Gortázar, C. \& Marco, J., 1998. Distribución de grandes mamíferos en la Comunidad Autónoma Aragonesa. Informe inédito del Gobierno de Aragón. 
Gortázar C., Herrero, J., Villafuerte, R. \& Marco, J., 2000. Historical examination of the status of large mammals in Aragon, Spain. Mammalia, 64: 411422.

Gortázar, C., Millán, J., Acevedo, P., Escudero, M. A., Marco, J. \& Fernández de Lucio, D., 2007. A large-scale survey of brown hare Lepus europaeus and Iberian hare Lepus granatensis population at the limit of their ranges. Wildlife Biology, 13 (3): 244-250.

Linell, J. D. C., Duncan, P. \& Andersen, R., 1998. The European roe deer: A portait of a successful species. The european roe deer: the biology of success. (R. Andersen, P. Duncan and J.D.C. Linnell editores): 11-22. Scandinavian University Press, Oslo (Noruega).

Marco, J., Herrero, J., Escudero, M. A., Fernández-Arberas, O., Ferreres, J., García-Serrano, A., Giménez-Anaya, A., Labarta, J. L., Monrabal, L. \& Prada, C., 2011. Veinte años de seguimiento poblacional de ungulados silvestres en Aragón. Pirineos, 166: 135-157.

Marco, J. \& Jordán, G., 1990. Estudio de la población de corzo en el Macizo del Moncayo y área de influencia. Informe interno del Gobierno de Aragón.

Mayle, B. A., Putman, R. J. \& Wyllie, I., 2000. The use of trackway counts to establish an index of deer presence. Mammal Review, 30: 233-237.

Mayle, B.A., Peace, J.A. \& Gill, R.M., 1999. How many deer? A field guide to estimating deer population size. UK: Forestry Commission. Field Book, 18: 93 pp. Edimburgo (Reino Unido).

Osuna, D., Prada, C., Herrero, J. \& Marco, J., 2006. Distribución de los ungulados silvestres en Aragón (2001-2005) determinada a partir de encuestas. Lucas Mallada, 13: 191-212.

Prada, C., García-Serrano, A., Fernández-Arberas, O. \& Herrero, J., 2010. Seguimiento del corzo Capreolus capreolus en el Pirineo aragonés (2002-2010). Informe interno del Gobierno de Aragón.

Putman, R., 2003. The deer manager's companion. Swan Hill Press, 180 pp. Bath. Reino Unido.

Sáez de Buruaga, M., 1997. La ordenación cinegética en caza mayor. I Curso de Gestión de Cotos. Fundación para el estudio y defensa de la naturaleza y de la caza mayor, 37-56. Madrid. España.

Sampietro, F. J., 2000. Descripción fisiográfica de la Comunidad Autónoma de Aragón. En: Aves de Aragón. Atlas de especies nidificantes. Diputación General de Aragón, segunda edición: 21-38. Zaragoza.

Sinclair, A.R., Fryxell, J. \& Caughley, G., 2006. Wilflife Ecology, Conservation, and Management. Blackwell Publishing: 450 pp. Oxford (Reino Unido).

Smart, J. C. R., Ward, A. I. \& White, P. C. L., 2004. Monitoring woodland deer populations in the UK: an imprecise science. Mammal Review, 34: 99-114. 
Strickland, M. D., Harju, H., McCaffert, K., Miller, H., Smith, L. \& Stoll, R., 1994. Harvest management. Research and management techniques for wildlife and habitats. (ed. T.A. Bookhout) ,445-473. The Wildlife Society, Bethesa, Md. Estados Unidos.

Tsaparis, D., Katsanevakis, S., Ntolka, E. \& Legakis, A., 2009. Estimating dung decay rates of roe deer (Capreolus capreolus) in different habitat types of a Mediterranean ecosystem: an information theory approach. European Journal of Wildife Research, 55: 167-172

Van Laere, G., Maillard, D., Boutin, J. M. \& Delorne, D., 1999. Le suivi des populations de chevreuils: des methodes traditionnelles d'estimation aux indicateurs population-enviroment. Bulletin Mensuel de l'Office National de la Chasse, $244: 46-53$.

Virgós, E. \& Tellería, J. L., 1998. Roe deer habitat selection in Spain: constraints on the distribution of a species. Canadian Journal of Zoology, 76: 1294-1299.

Williams, D., Acevedo, P., Gortázar, C., Escudero, M. A., Labarta, J. L., Marco, J. \& Villafuerte, R., 2007. Hunting for answers: rabbit (Oryctolagus ciniculus) population trends in northeastern Spain. Journal of Wildlife Research, 53: 19-28. 